\title{
Rotation of Simple Organic Systems Can Be Induced by Low Intensity Electromagnetic Fields
}

\author{
Emanuele Calabrò ${ }^{1,6 *}$ and Salvatore Magazù ${ }^{2-6}$ \\ ${ }^{1}$ Ministry of Instruction, University and Research (MIUR), Italy - Technological Institute of Messina "Verona Trento-Marconi", \\ 98123 Messina, Italy \\ ${ }^{2}$ Department of Mathematical and Informatics Sciences, Physical Sciences and Earth Sciences of Messina University, Viale Stagno D' \\ Alcontres 31, 98166 Messina, Italy \\ ${ }^{3}$ Le Studium, Loire Valley Institute for Advanced Studies, Orléans \& Tours, France \\ ${ }^{4}$ Centre de Biophysique Moleculaire (CBM)-CNRS UPR 4301 du CNRS, rue Charles Sadron, 45071 Orleans CEDEX 2 France; Laboratoire \\ Interfaces, Confinement, Matériaux et Nanostructures (ICMN) - UMR 7374 CNRS - Université d'Orléans, 1 b rue de la Férollerie, CS 40059, \\ 45071 Orléans cedex 2, France \\ ${ }^{5}$ Istituto Nazionale di Alta Matematica "F. Severi" - INDAM - Gruppo Nazionale per la Fisica Matematica - GNFM, Piazzale Aldo Moro, \\ 500185 Rome, Italy \\ ${ }^{6}$ CISFA "Interuniversity Consortium of Applied Physical Sciences" (Consorzio Interuniversitario di Scienze Fisiche Applicate), Viale \\ Ferdinando Stagno D' Alcontres 31, 98166 Messina, Italy \\ *Corresponding author: Emanuele Calabrò, Ministry of Instruction, University and Research (MIUR), Italy - Technological Institute \\ of Messina "Verona Trento-Marconi", 98123 Messina, Italy; CISFA "Interuniversity Consortium of Applied Physical Sciences" \\ (Consorzio Interuniversitario di Scienze Fisiche Applicate), Viale Ferdinando Stagno D’ Alcontres 31, 98166 Messina, Italy, \\ Email ID: e.calabro@yahoo.com
}

\section{ARTICLE INFO}

Received: 幽 September 01, 2021

Published: September 07, 2021

\section{ABSTRACT}

Citation: Emanuele Calabrò, Salvatore Magazù. Rotation of Simple Organic Systems Can Be Induced by Low Intensity Electromagnetic Fields. Biomed J Sci \& Tech Res 38(4)2021. BJSTR. MS.ID.006176.

\section{Mini Review}

Biological systems have magnetic properties due to microscopic atomic dipoles in their compounds which align themselves individually if a magnetic field is applied. As a result, a biological system can align along the direction of an applied magnetic field following the theory of paramagnetism. This effect can be induced also in simple organic systems. For instance, the $\alpha$-helix is the main structure of a protein. We should not be surprised by this effect as proteins $\alpha$-helix has generally a relevant dipole moment which allows possible orientation of a protein along the direction of an applied electromagnetic field. For instance, hemoglobin has a dipole moment of $280 \mathrm{D}$ [1]. Instead, what makes us wonder is the fact that this effect occurs even at very small intensity of an electromagnetic field regardless of its frequency. In order that this effect can be highlighted a sophisticated technique should be used, that is Fourier Transform Infrared (FTIR) spectroscopy.

FTIR spectroscopy can be considered as a valuable tool for analyzing the structure of proteins or other simple organic systems in aqueous solutions [2,3]. The use of this technique has evidenced a significant increase in intensity of proteins Amide I and Amide II vibration bands after exposure of typical proteins in water solutions to an electromagnetic field at a low intensity at $100 \mathrm{~mW} /$ $\mathrm{m} 2$ in the range $0.9-2.5 \mathrm{GHz}$ [4-7]. These vibration bands are characteristic of proteins secondary structure and are due above all to the $\alpha$-helix content. Hence, an increasing of Amide I and II bands can be explained assuming that proteins $\alpha$-helix aligned along the direction of applied electromagnetic field inducing an increasing 
of total amount of dipole moment. Typical proteins in bidistilled water solution were used in these experiments [4-6] in order to schematize cellular environment in which they are embedded. This effect was also observed in typical human cells [8-13]. Even this result should not surprise us because proteins $\alpha$-helices are present in all types of cellular membrane channels [14-16]. In particular, cellular membrane protein accounting for about $50 \%$ of its mass forming the wall of cells channels [17].

Rotation induced by exposure to magnetic fields was also observed in DNA and chromosomes in neuronal-like cells. Indeed, significant decrease in intensity of the phosphate bands in the DNA infrared region was observed by FTIR spectroscopy after exposure of human neuronal-like cells to static and $50 \mathrm{~Hz}$ magnetic field at the low-intensity value of $1 \mathrm{mT}$ [18]. This result can be explained assuming that uncoiling and unpackaging of chromatin constituents in chromosomes occurred after exposure, due to the torque induced by the applied magnetic field and the consequent chromosome alignment towards the direction of the field. Incidentally, the rotation of chromosomes produced by exposure to a magnetic field was observed directly by microscope of some plants, Allium sativum and Vicia faba, exposed to a magnetic field [18]. Also, this result depends on paramagnetic property of DNA constituents. However, these results seem that cannot be explained theoretically. In fact, Adair showed that the torque of an electromagnetic field at high intensity is lower than the mean angular momentum due to thermal molecular agitation in organic matter [19].

Nevertheless, it was shown that the impulse from an electromagnetic field integrated over an interval of some hour will overhead the mean angular momentum from thermal agitation [20], demonstrating that the torque induced by high frequency electromagnetic field from wireless devices can affect organic matter due to the duration of exposure. Indeed, the simulation of Adair can be applied to a macrodipole exposed to an electromagnetic field provided that the viscous damper of the medium in which it is embedded can be neglected, for instance in the vacuum. In contrast, organic systems are embedded in aqueous solutions with viscous damper that cannot be neglected. As a result, the macrodipole cannot follow the oscillation of the electromagnetic field due to the inertia of molecules and the viscosity of the medium, so that it will place itself at an average position along the direction of the field [7]. Hence, the entire exposure time should be taken into account in the calculation of the torque induced by an applied electromagnetic field so that the simulation is in agreement with experimental results.

\section{References}

1. Antosiewicz J, Porschke D (1995) Electrostatics of hemoglobins from measurements of the electric dichroism and computer simulations. Biophys J 68(2): 655-664.

2. Byler DM, Susi H (1986) Examination of the secondary structure of proteins by deconvolved FTIR spectra. Biopolymer 25(3): 469-487.
3. Surewicz WK, Mantsch HH (1988) New insight into protein secondary structure from resolution-enhanced infrared spectra. Biochim Biophys Acta 952(2): 115-130

4. Calabrò E, Magazù S (2016) Parallel $\beta$-sheet Vibration Band Increases with Proteins Dipole Moment under Exposure to $1765 \mathrm{MHz}$ Microwaves. Bioelectromagnetics 37(2): 99-107.

5. Calabrò E, Magazù S (2017) The $\alpha$-Helix Alignment of Proteins in Water Solution towards a High Frequency Electromagnetic Field: a FTIR Spectroscopy Study. Electromagnetic Biology and Medicine 36(3): 279288.

6. Calabrò E, Magazù S, Campo S (2012) Microwave-induced increase of amide I and amide II vibration bands and modulating functions of sodium-chloride, sucrose and trehalose aqueous solutions: The case study of Haemoglobin. Research Journal of Chemistry and Environment 16(4): 59-67.

7. Calabrò E, Magazù S (2018) Resonant interaction between electromagnetic fields and proteins: A possible starting point for the treatment of cancer. Electromagnetic Biology and Medicine 37(3): 155168.

8. Calabrò E, Condello S, Currò M, Ferlazzo N, Vecchio M, et al. (2013) $50 \mathrm{~Hz}$ Electromagnetic Field Produced Changes in FTIR Spectroscopy Associated with Mitochondrial Transmembrane Potential Reduction in Neuronal-Like SH-SY5Y Cells. Oxidative Medicine and Cellular Longevity 2013: 414393.

9. Calabrò E (2016) Competition between Hydrogen Bonding and Protein Aggregation in Neuronal-Like Cells under Exposure to $50 \mathrm{~Hz}$ Magnetic Field. International Journal of Radiation Biology 92(7): 395-403.

10. Calabrò E, Magazù S (2019) New Perspectives in the Treatment of Tumor Cells by Electromagnetic Radiation at Resonance Frequencies in Cellular Membrane Channels. The Open Biotechnology Journal 13: 105-110.

11. Calabrò E, Magazù S (2019) Infrared Spectroscopic Demonstration of Magnetic Orientation in SH-SY5Y Neuronal-Like Cells Induced by Static or $50 \mathrm{~Hz}$ Magnetic Fields. International Journal of Radiation Biology 95(6): 781-787

12. Calabrò E, Currò M, Caccamo MT (2020) Competition between N-H bending vibration and $\alpha$-helix polarization under $50 \mathrm{~Hz}$ magnetic field in SH-SY5Y neuronal-like cells. Spectroscopy Letters: An International Journal for Rapid Communication 53(6): 458-465.

13. Calabrò E, Magazù S (2020) Applying Resonant Electromagnetic Fields in Membrane Channels of Tumor Cells: The Future of Cancer Treatment. Biomed J Sci \& Tech Res 31(4): 2020.

14. Becker L, Bannwarth M, Meisinger C (2005) Preprotein translocase of the outer mitochondrial membrane: Reconstituted Tom 40 forms a characteristic TOM pore. J Mol Biol 353: 1011-1020.

15. Feria Bourrellier AB, Valot B, Guillot A (2010) Chloroplast acetyl-CoA carboxylase activity is 2-oxoglutarate-regulated by interaction of PII with the biotin carboxyl carrier subunit. Proc Natl Acad Sci USA 107: 502-507.

16. Szabo I, Zoratti M (2014) Mitochondrial channels: Ion fluxes and more. Physiol Rev 94: 519-608.

17. Alberts B, Johnson A, Lewis J (2002) Molecular Biology of the Cell (4 $4^{\text {th }}$ Edn.)., Garland Science.

18. Calabrò E, Goswami HK, Magazù S (2020) Chromosome aberration in typical biological systems under exposure to low- and high intensity magnetic fields. Electromagnetic Biology and Medicine 39(2): 97-108.

19. Adair RK (2000) Biophysics Limits on the Biological Effects of Ultrawide band Electromagnetic Radiation. Springer.

20. Calabrò E, Magazù S (2018) Direct spectroscopic evidence for competition between thermal molecular agitation and magnetic field in a tetrameric protein in aqueous solution. Physics Letters A 382: 13891394. 


\section{ISSN: 2574-1241}

DOI: $10.26717 /$ BJSTR.2021.38.006176

Emanuele Calabrò. Biomed J Sci \& Tech Res

(C) (P) This work is licensed under Creative

Submission Link: https://biomedres.us/submit-manuscript.php

$\begin{array}{ll}\text { BIOMEDICAL } & \text { Assets of Publishing with us } \\ \text { RESEARCHES } & \text { Global archiving of articles } \\ \text { - Immediate, unrestricted online access }\end{array}$

